\title{
How can we fight against antimicrobial- resistant bacteria in the World Health Organization Western Pacific Region?
}

\author{
Yoshiaki Gu and Mitsuo Kakub \\ Correspondence to Yoshiaki Gu (e-mail: ygu@med.tohoku.ac.jp)
}

$\mathrm{T}$ he public health community is faced with the global challenge posed by antimicrobial-resistant bacteria, including meticillin-resistant Staphylococcus aureus (MRSA) and extended-spectrum $\beta$-lactamases (ESBLs)-producing enterobacteriaceae. ${ }^{1}$ The need to address this issue prompted the focus of World Health Day 2011, which was entitled "Antimicrobial resistance: no action today, no cure tomorrow."

In today's world of international travel, the globalization of drug-resistant bacteria is a pressing issue for public health professionals. In the World Health Organization (WHO) Western Pacific Region, as well as other regions in the world, more and more new types of antimicrobial-resistant bacteria have come to the forefront. An important example is New Delhi Metallo$\beta$-lactamase 1 (NDM-1)-carrying enterobacteriaceae, which attracted attention in Europe in 2010 as imported cases associated with health care contact in India and Bangladesh. Although some NDM-1 cases were reported from Australia and Japan, ${ }^{2}$ no outbreaks were reported in the Western Pacific Region.

In this perspective article, we consider four focus areas for countries in the Western Pacific Region to consider when strategizing their response to antimicrobial resistant bacteria.

\section{(1) Surveillance of resistant bacteria}

There is no formal framework for collaboration among surveillance programmes on antimicrobial resistance worldwide. $^{3}$ Some countries in the WHO Western Pacific Region have national surveillance systems (e.g. Japan and Hong Kong [China]). The WHO Western Pacific Regional Office conducted the Regional Programme for Surveillance of antimicrobial resistance from 1990 to 2000 and has recently established a new Working Group which has identified surveillance of resistance as a regional priority. ${ }^{3}$ According to the WHO Western Pacific Regional Office website, it annually accumulates drug resistance data from 14 focal laboratories in 13 countries based on their surveillance systems ${ }^{4}$ however, no data is published on its website at the moment.

Constructing a standardized surveillance system in the Region would provide useful data to monitor and assess the pattern and frequency of resistant bacteria. Each country should establish and strengthen its reference laboratory and national surveillance programme. Building up standard methods and quality analyses in laboratories by using quality assessment schemes is crucial to establish multi-laboratory networks. WHONET, a free database software for management and analysis of microbiology laboratory data, would allow some measures of standardization. The surveillance system in European countries (European Antimicrobial Resistance Surveillance Network [EARS-Net]) is a useful model to enhance the international surveillance network among countries in the Western Pacific Region. Such a network would assist countries, especially when dealing with cross-border outbreaks of resistant bacteria. The WHO South-East Asia and the Western Pacific Regional Offices are collaborating to build up standard laboratory methods and surveillance systems to monitor resistant bacteria in both regions.

\section{(2) Basic research of resistant bacteria}

Basic research of drug-resistant bacteria, such as identifying responsible genes and enzymes, is crucial to

\footnotetext{
Department of Regional Cooperation for Infectious Diseases, Tohoku University Graduate School of Medicine, Sendai, Japan

Department of Infection Control and Laboratory Diagnosis, Tohoku University Graduate School of Medicine, Sendai, Japan.

Submitted: 29 November 2011 ; Published: 30 July 2012

doi: 10.5365/wpsar.2011.2.4.017
} 
understanding the mechanism of antimicrobial resistance and transmission. Among gram-positive bacteria, clonal spreading of community-associated MRSA (CA-MRSA) is an important topic. Staphylococcus aureus is one of the leading causes of bacterial infection worldwide. CA-MRSA have a different genetic profile to that of health-care-associated MRSA (HA-MRSA), yet are more virulent and transmissible than HA-MRSA. ${ }^{5}$ CA-MRSA infections are epidemic in some countries including Asian countries. ${ }^{6}$ Molecular typing (e.g. multilocus sequence typing [MLST]) provides information on distribution patterns that may translate to transmission routes of MRSA in communities. For instance, a combination of epidemiological, bacteriological and molecular methods showed the transmission tree and support control measures of an animal-origin MRSA epidemic in the Netherlands. ${ }^{7}$ Among gram-negative bacteria, ESBLproducing enterobacteriaceae is a pressing topic in the Western Pacific Region. ${ }^{8}$ The mechanisms of carbapenem resistance in enterobacteriaceae, Pseudomonas aeruginosa and Acinetobacter baumannii are also receiving attention. Our group is trying to determine the distribution pattern of carbapenem-resistant Acinetobacter baumannii by using MLST. ${ }^{9}$

\section{(3) Appropriate use of antimicrobials}

Overuse and misuse of antimicrobials are considered to be major causes of the increase of drug-resistant bacteria. ${ }^{1}$ Antimicrobial stewardship is a key component of a multifaceted approach to prevent emergence of antimicrobial resistance. Antimicrobial stewardship includes optimizing antimicrobial selection, dosing, routes and duration of therapy as well as limiting inappropriate antimicrobial use.

Detecting trends of antimicrobial consumption is necessary to estimate resistant selective pressure and enhance antimicrobial management in hospitals and communities. Antimicrobial usage is one of the major intervention points on antimicrobial resistance issues. Although there is no comprehensive surveillance systems of antimicrobial consumption in the Western Pacific Region, the European Surveillance of Antimicrobial Consumption (ESAC) collects data from either distribution or reimbursement systems in each country. ${ }^{10}$ A combination of surveillance of resistance and antimicrobials in the Western Pacific Region would be helpful to evaluate the association between antimicrobial usage and the trend of resistance.
Non-prescription antimicrobial use is frequent in some countries, especially in developing countries, and is considered a cause of antimicrobial resistance. According to surveys from Asian countries including China, the Philippines and Viet Nam, more than half of antimicrobials used were non-prescribed drugs. ${ }^{11}$ There is also great concern about the poor quality of antimicrobials mostly in developing countries. Counterfeit, substandard or degraded antimicrobials are likely to worsen drug-resistance. Although counterfeit drugs with no active ingredient will not select drug resistant bacteria, those containing the wrong active ingredients with antibacterial effects may affect the emergence and spread of drug-resistant bacteria.

Antimicrobial use in livestock and fishery industries are also considered to have a potential impact on resistant bacteria among humans. Fluoroquinoloneresistant Campylobacter species in humans, for instance, are associated with fluoroquinolone use in poultry. ${ }^{12}$ It is important to follow the harmful effect of these kinds of irregular antimicrobial uses on resistant bacteria. Tackling antimicrobial resistance requires an intersectional approach with effective coordination of action and an exchange of information among food, veterinary and health sectors in each country and relevant international organizations. Enhancement of collaboration between veterinary and human medicine would accelerate interdisciplinary and international action.

\section{(4) Infection prevention and control practices}

Basic infection prevention and control practices are essential to prevent the spread of drug-resistant bacteria in medical facilities. Standard precautions, including basic hand hygiene with soap and water or alcohol, are the most basic practices in the care of all patients. Contact precautions are recommended in the care of patients with antimicrobial resistant bacteria. ${ }^{13}$ Although these are crucial in both developed and developing countries, the risk of health care-associated infections seems to be higher in developing countries. Medical systems in developing countries are not always advanced enough to implement sufficient infection control practices.

WHO has published guidelines on hand hygiene in health care settings, how to organize training programmes and how to establish good practices among health care workers. ${ }^{14}$ The WHO Western Pacific Regional Office can work with countries to step up their skills and resources 
to protect patients in medical facilities and provide appropriate support.

Each country in the Western Pacific Region should act now to prevent the increase and spread of antimicrobial resistant bacteria. WHO should provide support for national and cross-border actions in the Western Pacific Region. Now is the time to develop a comprehensive strategy using the four focus areas of this perspective article to effectively combat antimicrobial resistance.

\section{Conflicts of interest}

None declared.

\section{Funding}

None.

\section{References:}

1. Lubelchek RJ, Weinstein RA. Antibiotic resistance and nosocomial infections. In: Mayer KH, Pizer HF, editors. The Social Ecology of Infectious Diseases. Massachusetts, Academic Press, 2008, 241-274.

2. Rogers BA et al. Country-to-country transfer of patients and the risk of multi-resistant bacterial infection. Clinical Infectious Diseases, 2011, 53:49-56. doi:10.1093/cid/cir273 pmid:21653302

3. Grundmann $\mathrm{H}$ et al. A framework for global surveillance of antibiotic resistance. Drug Resistance Updates, 2011, 14:79-87. doi:10.1016/j.drup.2011.02.007 pmid:21482177

4. Emerging disease surveillance and response. Surveillance, Network on antimicrobial resistance. Manila, World Health Organization Western Pacific Regional Office, 2012 (http://www.wpro.who. int/entity/emerging diseases/Surveillance/en/index.html, accessed 15 June 2012).
5. Deleo FR et al. Community-associated meticillin-resistant Staphylococcus aureus. Lancet, 2010, 375:1557-1568. doi:10.1016/S0140-6736(09)61999-1 pmid:20206987

6. Song JH et al, ANSORP Study Group. Spread of methicillin-resistant Staphylococcus aureus between the community and the hospitals in Asian countries: an ANSORP study. The Journal of Antimicrobial Chemotherapy, 2011, 66:1061-1069. doi:10.1093/jac/dkr024 pmid:21393157

7. van Loo I et al. Emergence of methicillin-resistant Staphylococcus aureus of animal origin in humans. Emerging Infectious Diseases, 2007, 13:1834-1839. doi:10.3201/eid1312.070384 pmid: 18258032

8. Hirakata $Y$ et al, SENTRY Asia-Pacific Participants. Regional variation in the prevalence of extended-spectrum beta-lactamaseproducing clinical isolates in the Asia-Pacific region (SENTRY 1998-2002). Diagnostic Microbiology and Infectious Disease, 2005, 52:323-329. doi:10.1016/j.diagmicrobio.2005.04.004 pmid: 16165001

9. Endo $S$ et al. Molecular epidemiology of carbapenem-nonsusceptible Acinetobacter baumannii in Japan. The Journal of Antimicrobial Chemotherapy, 2012, 67(7):1623-1626. doi:10.1093/jac/dks094 pmid:22447879

10. Vander Stichele RH et al, ESAC Project Group. European surveillance of antimicrobial consumption (ESAC): data collection performance and methodological approach. British Journal of Clinical Pharmacology, 2004, 58:419-428. doi:10.1111/ j.1365-2125.2004.02164.x pmid:15373935

11. Morgan DJ et al. Non-prescription antimicrobial use worldwide: a systematic review. The Lancet Infectious Diseases, 2011, 11:692-701. doi:10.1016/S1473-3099(11)70054-8 pmid:21659004

12. Nelson JM et al. Fluoroquinolone-resistant Campylobacter species and the withdrawal of fluoroquinolones from use in poultry: a public health success story. Clinical Infectious Diseases, 2007, 44:977-980. doi:10.1086/512369 pmid:17342653

13. Siegel JD et al, Health Care Infection Control Practices Advisory Committee. 2007 Guideline for Isolation Precautions: Preventing Transmission of Infectious Agents in Health Care Settings. American Journal of Infection Control, 2007, 35 Suppl 2:S65164. doi:10.1016/j.ajic.2007.10.007 pmid:18068815

14. Guidelines on Hand Hygiene in Health Care. Geneva, World Health Organization, 2012 (http://whqlibdoc. who.int/publications/2009/9789241597906 eng.pdf, accessed 15 June 2012). 